\section{Mucormycosis May Mimic Disease Relapse in Wegener's Granulomatosis}

\section{To the Editor:}

Mucormycosis is a life-threatening infection that occurs predominantly in diabetic or immunosuppressed patients. Successful treatment requires early diagnosis, prompt antifungal therapy, and surgical debridement. We describe a patient with severe relapsing Wegener's granulomatosis (WG), with pulmonary mucormycosis mimicking vasculitic disease relapse, successfully treated with combined posaconazole and amphotericin.

A 29-year-old man was diagnosed with WG on the basis of a lung biopsy and a positive antineutrophil cytoplasm antibody (ANCA), who had presented with lung hemorrhage and transient acute renal failure. Plasmapheresis, steroids, and cyclophosphamide were administered, with complete recovery of renal and pulmonary function. He was maintained on azathioprine and prednisolone for 3 years, but subsequently developed a relapsing-remitting disease pattern. Over 2 years he had 4 relapses, with sinusitis, pulmonary nodules, and subglottic stenosis. He was initially treated with mycophenolate mofetil and prednisolone, while subsequent relapses required use of cyclophosphamide, infliximab, and finally rituximab. This resulted in successful disease remission and he was again maintained on low-dose azathioprine and prednisolone. He remained persistently ANCA-positive throughout his followup.

Seven years after his initial presentation, he reported 2 weeks of fevers, night sweats, cough, hemoptysis, pleuritic chest pain, and exertional dyspnea. Of note, his house had recently been renovated. Examination was unremarkable except for fever and widespread crackles in both lung fields. There were elevated inflammatory markers [white blood cell count $13.1 \times$ $10^{9} / 1$; platelets $472 \times 10^{9} / 1$; C-reactive protein $\left.(\mathrm{CRP}) 100 \mathrm{mg} / \mathrm{dl}\right]$ and an elevated PR3-ANCA titer of $903 \mathrm{IU} / \mathrm{ml}$ (normal 0-25), which had risen from $491 \mathrm{IU} / \mathrm{ml} 3$ months earlier. Repeated blood cultures and viral serology (for respiratory syncitial virus, influenza A and B, parainfluenza 1, 2 and 3 , and adenovirus) were all negative. His peripheral $\mathrm{B}$ cells had reconstituted (absolute B lymphocyte count 104 B cells/ $\mu 1$; normal 100-400). He was not diabetic.

High resolution chest computed tomography (HRCT; Figure 1) showed

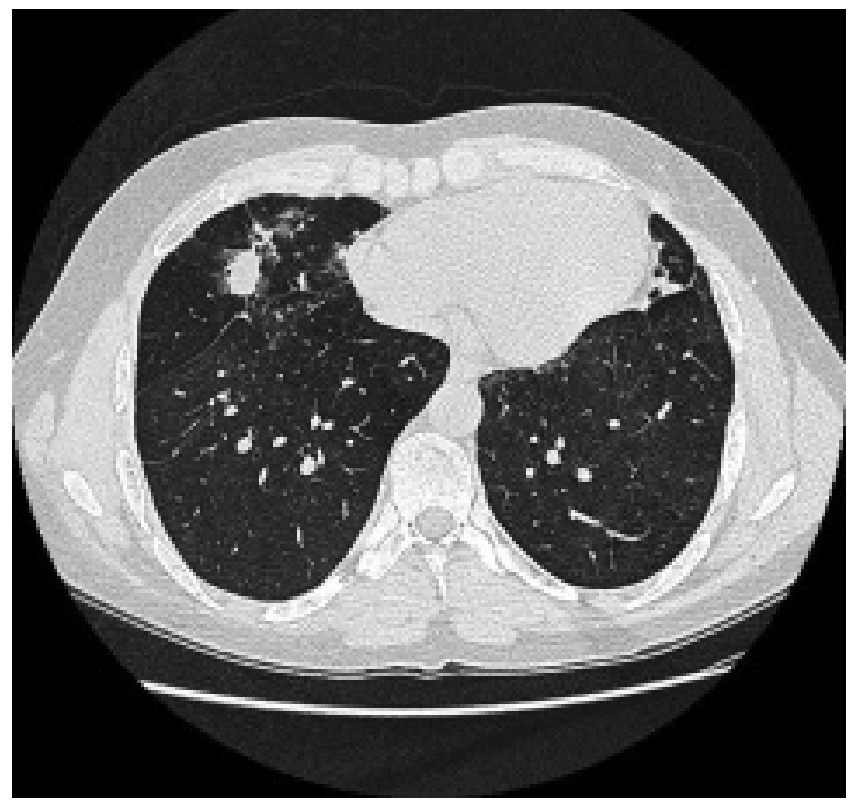

Figure 1. High resolution CT of thorax. A $2 \mathrm{~cm} \times 1.4 \mathrm{~cm}$ soft-tissue nodule is present within the middle lobe of right lung with minimal surrounding inflammatory ground-glass changes. There are adjacent longstanding fibrotic lung changes. In addition, traction bronchiectasis in the upper lobes and aortopulmonary lymphadenopathy were noted. new soft-tissue lesions within the lingula and right middle and lower lobes, with associated ground-glass change. Traction bronchiectasis and aortopulmonary lymphadenopathy were also observed. Sinus CT revealed no abnormality.

Together these features were highly suggestive of active vasculitic relapse and he was considered for repeat rituximab dosing. However, since he had been exposed to prolonged immunotherapy, he underwent bronchoscopy and bronchoalveolar lavage (BAL) to exclude infection. BAL fluid grew Rhizopus arrhizus, a commonly isolated agent of mucormycosis, and scanty Candida albicans. The Rhizopus was resistant to flucytosine and fluconazole (MIC > 32), but sensitive to amphotericin (MIC 0.5).

Liposomal amphotericin B was started. Concerns regarding the aggressive nature of mucormycosis and the need to maintain immunosuppressive therapy to avoid relapse of vasculitis led to the addition of posaconazole. The patient responded clinically with reduction in his CRP and PR3-ANCA (Figure 2), despite immunosuppression with the same doses of azathioprine and steroids. Repeat HRCT after 7 weeks of posaconazole therapy showed regression of the previous lung lesions. After 2 years of followup he remains well.

Mucormycosis is a frequently lethal opportunistic infection caused by fungi of the order Mucorale in the class Zygomycetes. Despite the ubiquitous expression of the fungus, infectious complications are rare, occurring mainly in immunocompromised patients and those with diabetes.

Airborne fungal spores reach the nasal mucosa or the alveoli where effective immunity is provided by neutrophils and macrophages ${ }^{1}$. When these mechanisms are disturbed, the fungi can become angioinvasive, leading to hematogenous spread, thrombosis, and infarction.

Mucormycosis generally presents with rhino-orbito-cerebral, pulmonary, or disseminated disease. Diagnosis relies upon identification of the agent in tissue or by culture. Management involves debridement of affected tissues and antifungal treatment. However, mortality rates remain high, at up to $87 \%$ in those with pulmonary disease ${ }^{2}$. To date, antifungal therapy has consisted mainly of amphotericin B; however, posaconazole has shown activity against Zygomycetes ${ }^{3}$.

Despite the presence of airway inflammation in patients with WG, and the use of immunosuppression, there is only one other case report of mucormycosis in a patient with systemic vasculitis ${ }^{4}$. This suggests that such infections may be underdiagnosed. Since the presentation may mimic a disease relapse so closely, it is possible that many such cases have been treated as vasculitic relapses that have responded poorly to increased immunosuppression. The changes in PR3-ANCA in relation to the fungal

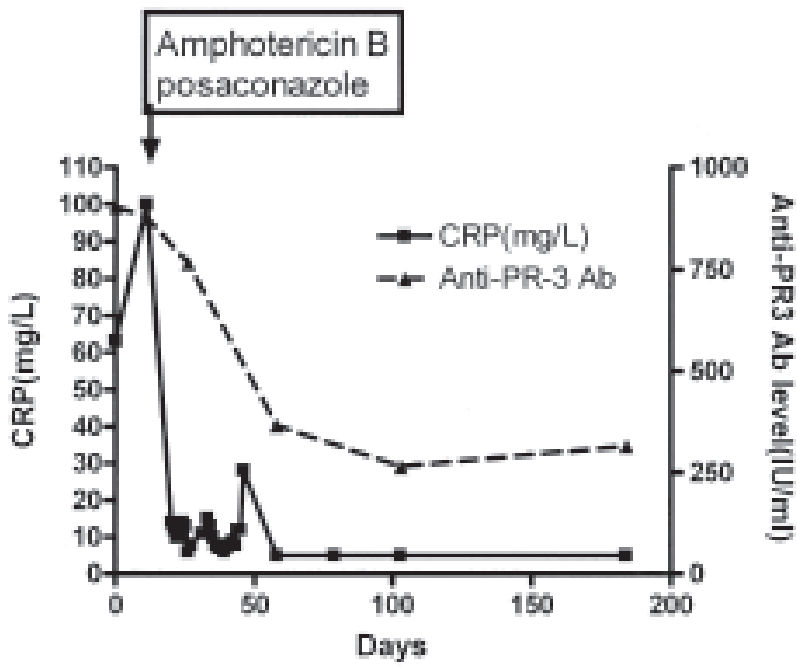

Figure 2. Changes in CRP and anti-PR3 antibody titers from the time of presentation with mucormycosis, during treatment with antifungal agents, and during followup. 
infection and its clearance may have been due to the well described association of infection stimulating an augmented autoimmune response. We suggest that, in heavily immunosuppressed patients with potential sinus or pulmonary relapse, careful consideration be given to excluding mucormycosis, by radiological and microbiological assessment, including lavage and culture of the affected organs.

ESTELA L. NOGUEIRA, MD, Centro Hospitalar de Vila Nova de Gaia, Porto, Portugal; PHILIP W. IND, BA, MA, MB, BChir, FRCP, Respiratory Section, Division of Medicine, Imperial College London, Hammersmith Hospital, London; JON S. FRIEDLAND, MA, PhD, FRCP, FRCPE, FMedSci, Department of Infectious Diseases and Immunity, Division of Medicine, Hammersmith Campus, Imperial College London; ALAN D. SALAMA, MA, PhD, FRCP, Renal Section, Division of Medicine, Imperial College London, Hammersmith Hospital, London, W12 0NN UK. Address correspondence to Dr. A.D. Salama; E-mail: a.salama@imperial.ac.uk We are grateful to Dr. Steven Moser for reporting the radiological findings and to Prof. Charles Pusey for his clinical input and assistance in writing the manuscript.

\section{REFERENCES}

1. Shoham S, Levitz SM. The immune response to fungal infections. Br J Haematol 2005;129:569-82.

2. Roden MM, Zaoutis TE, Buchanan WL, Knudsen TA, Sarkisova TA, Schaufele RL, et al. Epidemiology and outcome of zygomycosis: a review of 929 reported cases. Clin Infect Dis 2005;41:634-53.

3. Petrikkos G, Skiada A. Recent advances in antifungal chemotherapy. Int J Antimicrob Agents 2007;30:108-17.

4. Berenguer J, Solera J, Moreno S, Munoz P, Parras F. Mucormycosis. The disease spectrum in 13 patients. Med Clin (Barc) 1990;94:766-72.

J Rheumatol 2010;37:6; doi:10.3899/jrheum.091423 